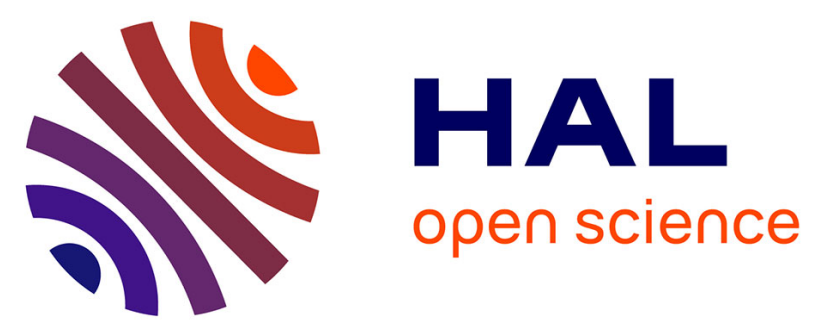

\title{
ATP binding cassette (ABC) transporters: expression and clinical value in glioblastoma
}

Antonin Dréan, Shai Rosenberg, François-Xavier Lejeune, Larissa Goli, Aravindan Arun Nadaradjane, Jeremy Guehennec, Charlotte Schmitt, Maïté Verreault, Franck Bielle, Karima Mokhtari, et al.

\section{To cite this version:}

Antonin Dréan, Shai Rosenberg, François-Xavier Lejeune, Larissa Goli, Aravindan Arun Nadaradjane, et al.. ATP binding cassette $(\mathrm{ABC})$ transporters: expression and clinical value in glioblastoma. Journal of Neuro-Oncology, In press, 10.1007/s11060-018-2819-3 . hal-01744606

\section{HAL Id: hal-01744606 https://hal.sorbonne-universite.fr/hal-01744606}

Submitted on 27 Mar 2018

HAL is a multi-disciplinary open access archive for the deposit and dissemination of scientific research documents, whether they are published or not. The documents may come from teaching and research institutions in France or abroad, or from public or private research centers.
L'archive ouverte pluridisciplinaire HAL, est destinée au dépôt et à la diffusion de documents scientifiques de niveau recherche, publiés ou non, émanant des établissements d'enseignement et de recherche français ou étrangers, des laboratoires publics ou privés. 


\section{ATP Binding Cassette (ABC) transporters: expression and clinical value in glioblastoma}

Dréan Antonin ${ }^{1,2}$, Rosenberg Shai ${ }^{1,3}$, Lejeune François-Xavier ${ }^{1}$, Goli Larissa ${ }^{1}$, Nadaradjane Aravindan Arun ${ }^{1}$, Guehennec Jérémy ${ }^{1}$, Schmitt Charlotte ${ }^{1}$, Verreault Maïté $^{1}$, Bielle Franck ${ }^{1,4,7}$, Mokhtari Karima ${ }^{1,4,7}$, Sanson Marc ${ }^{1,5,7}$, Carpentier Alexandre $^{6}$, Delattre Jean-Yves ${ }^{1,5,7}$, Idbaih Ahmed ${ }^{1,5}$

1 Inserm U 1127, CNRS UMR 7225, Sorbonne Universités, UPMC Univ Paris 04 UMR S 1127, Institut du Cerveau et de la Moelle épinière, ICM, F-75013, Paris, France

2 Equipe de recherche CarThera, Institut du Cerveau et de la Moelle épinière, Ipeps ICM, F-75013, Paris, France.

3 Hadassah - Hebrew University Medical Center, Israel (S.R.)

4 AP-HP, Hôpitaux Universitaires La Pitié Salpêtrière - Charles Foix, Service de Neuropathologie, F-75013, Paris, France.

5 AP-HP, Hôpitaux Universitaires La Pitié Salpêtrière - Charles Foix, Service de Neurologie 2-Mazarin, F-75013, Paris, France.

6 AP-HP, Hôpitaux Universitaires La Pitié Salpêtrière - Charles Foix, Service de Neurochirurgie, F-75013, Paris, France.

7 OncoNeuroTek, Hôpitaux Universitaires La Pitié Salpêtrière, Paris, France 


\section{Corresponding author}

Ahmed Idbaih. Hôpitaux Universitaires La Pitié Salpêtrière - Charles Foix, Service de Neurologie 2-Mazarin, F-75013, Paris, France. Tél: 0033142140385. Fax 00331 42140418. Email ahmed.idbaih@aphp.fr or ahmed.idbaih@gmail.com 


\section{Abstract}

ATP-binding cassette transporters (ABC transporters) regulate traffic of multiple compounds, including chemotherapeutic agents, through biological membranes. They are expressed by multiple cell types and have been implicated in the drug resistance of some cancer cells. Despite significant research in $A B C$ transporters in the context of many diseases, little is known about their expression and clinical value in glioblastoma (GBM).

We analyzed expression of $49 \mathrm{ABC}$ transporters in both commercial and patientderived GBM cell lines as well as from 51 human GBM tumor biopsies. Using The Cancer Genome Atlas (TCGA) cohort as a training dataset and our cohort as a validation dataset, we also investigated the prognostic value of these $A B C$ transporters in newly diagnosed GBM patients, treated with the standard of care.

In contrast to commercial GBM cell lines, GBM-patient derived cell lines (PDCL), grown as neurospheres in a serum-free medium, express $A B C$ transporters similarly to parental tumors. Serum appeared to slightly increase resistance to temozolomide correlating with a tendency for an increased expression of $A B C B 1$. Some differences were observed mainly due to expression of $A B C$ transporters by microenvironmental cells. Together, our data suggest that the efficacy of chemotherapeutic agents may be misestimated in vitro if they are the targets of efflux pumps whose expression can be modulated by serum. Interestingly, several $A B C$ transporters have prognostic value in the TCGA dataset. In our cohort of 51 GBM patients treated with radiation therapy with concurrent and adjuvant temozolomide, ABCA13 overexpression is associated with a decreased progression free survival in univariate $(p<0.01)$ and multivariate analyses including MGMT promoter methylation ( $p=0.05)$ suggesting reduced sensitivity to temozolomide in ABCA13 overexpressing GBM. 
Expression of $\mathrm{ABC}$ transporters is: (i) detected in GBM and microenvironmental cells and (ii) better reproduced in GBM-PDCL. ABCA13 expression is an independent prognostic factor in newly diagnosed GBM patients.

Further prospective studies are warranted to investigate whether $A B C A 13$ expression can be used to further personalize treatments for GBM.

Keyword: Glioblastoma, resistance, $A B C$ transporters, PDCL, $A B C A 13$ 


\section{Introduction}

Glioblastoma (GBM) is the most common and aggressive primary brain cancer in adults, with a median overall survival below 20 months after the initial diagnosis [1]. Virtually, all GBM patients experience tumor relapse after first-line standard of care. The blood-brain barrier (BBB), a biophysical barrier limiting penetration of chemotherapeutic agents within the brain and the brain tumor, is one of the actors involved in intrinsic resistance of drugs in GBM patients [2].

ATP binding cassette $(A B C)$ transporters are a family of 49 proteins that actively transport many substrates. This family contains the main efflux pumps that expel drugs out of cells, therefore impacting chemosensitivity [3]. Efflux pumps are expressed by many cells in the body. In the brain, they are expressed by microglial cells, astrocytes, neurons, pericytes and, endothelial cells [4]. Endothelial cells, which are involved in the normal functioning of the BBB, mainly express ABCB1 (P-gp) and ABCG2 (BCRP) to efflux xenobiotics from the brain parenchyma to the bloodstream [2]. In addition, many $A B C$ transporters are involved in cell metabolism and/or transport of biologic molecules [3].

$A B C$ transporters have a large spectrum of substrates, including many chemotherapeutic agents [5]. The efflux of these therapeutic agents from the brain to the blood by the BBB hampers their access to tumor cells and their efficacy. Efflux pumps, from the $A B C$ transporter family, can also be expressed by tumor cells themselves, leading to the development of intrinsic chemoresistance [6].

Here the expression of all $A B C$ transporters in GBM cells and their microenvironment was assessed and their potential prognostic impact in a homogeneous population of GBM patients was further investigated. 


\section{Material and methods}

Public dataset

Levels of mRNA expression of the $49 \mathrm{ABC}$ transporters were downloaded from The Cancer Genome Atlas (TCGA) GBM dataset [7]. The threshold of z-scores (RNA Seq V2 RSEM) was set at \pm 2 of distinguishing over- and under-expressing samples. Overall survival (OS; from diagnosis to death) and progression-free survival (PFS; from diagnosis to first tumor progression) were compared using log-rank test. Pvalues below 0.05 were considered significant. RNAseq data for the U251 cell line were obtained from Lundberg et al. [8]. Expression levels of ABCB1, ABCG1 and ABCG2 by normal CNS cells were obtained from http://web.stanford.edu/group/barres_lab/brain_rnaseq.html [9].

OncoNeuroTek tissue and data as validation cohort

For the validation, patients and tumors from the OncoNeuroTek tissue bank were selected according to the following inclusion criteria : (i) newly diagnosed and histologically proven GBM, (ii) age below 70 years, (iii) Karnofsky performance status above $70 \%$, (iv) treated with radiation therapy with concurrent and adjuvant temozolomide [10], (v) available MGMT promoter methylation status, (vi) available clinical data, (vii) available tumor tissue for additional molecular analysis and, (viii) signed informed consent form for molecular analysis.

GBM cell lines

Nine GBM-patient derived cell lines (PDCL) named 4339, 4371, 5706, 6190, 6240, $7015,7060,7142$ and N13-1520 were established in our laboratory. Tissue from the 
paired human tumor samples was available for further molecular analysis. These samples and associated clinico-molecular annotations were previously reported [11].

\section{Cell culture}

Cell cultures were performed as previously described [11]. GBM-PDCL were established (passage $>$ 5) and cultured in DMEM-F12 (Life Technologies, 31331028) supplemented with $1 \%$ penicillin/streptomycin, B27 supplement 50X (Life Technologies, 17504-044), $20 \mathrm{ng} / \mathrm{mL}$ human bFGF (Peprotech, 100-18B) and 20 ng/ml human EGF (Peprotech, 100-15). Cells were cultured as gliomaspheres. Dissociation was performed with Accutase StemPro (Life Technologies, A11105-11) after washing with HBSS (Life Technologies, 1150029). For culture in serum, PDCL were cultured in DMEM (Life Technologies,31885049) supplemented with 10\% FBS (Thermo Fisher Life) and 1\% penicillin/streptomycin.

Viability test.

For drug sensitivity assays, 96-well plates were used. For viability assay on PDCL, wells were coated with $10 \mu \mathrm{g} / \mathrm{mL}$ laminin (cat no. L2020, Sigma-Aldrich) at $37^{\circ} \mathrm{C}$ for 1 hour for PDCL. Six thousand cells were plated per well. Temozolomide (SigmaAldrich, T2577) was resuspended in DMSO as a $20 \mathrm{mg} / \mathrm{ml}$ stock solution and was added 24 hours after plating. 150 hours after drug addition, WST-1 reagent (Roche) was added according to the manufacturer's instructions. WST-1 salt is cleaved to a soluble formazan dye by a $\mathrm{NAD}(\mathrm{P}) \mathrm{H}$-dependent reaction in viable cells. Plates were incubated for 3 hours and read by spectrophotometry at $450 \mathrm{~nm}$ and $620 \mathrm{~nm}$ wavelength. 


\section{RNA extraction}

RNA samples from the 9 GBM tumors and their 9 paired GBM-PDCL were provided by OncoNeuroTek tissue bank. RNA extraction for tumor samples was performed as previously described using the RNeasy Lipid Tissue Mini Kit (Qiagen) [12].

For GBM-PDCL, $1 \times 10^{4}$ dissociated cells were cultured in a $75 \mathrm{~cm}^{2}$ flask for four days, with culture medium changed at day 3 , and cells were harvested the next day as a dry pellet and stored at $-80^{\circ} \mathrm{C}$ as described previously [11]. RNA extraction was performed using the kit NucleoSpin ${ }^{\circledR}$ RNA (Macherey-Nagel, 740955) according the manufacturer's guidelines.

\section{Reverse Transcription and Real-Time Quantitative PCR (RT-qPCR)}

Reverse transcription was performed using the kit Maxima First Strand cDNA Synthesis Kit for RT-qPCR (ThermoScientific, K1442) according to the manufacturer's guidelines starting with $750 \mathrm{ng}$ of RNA. cDNAs were stored at $-20^{\circ} \mathrm{C}$. qPCR was used to examine expression levels of $A B C A 13, A B C B 1, A B C G 1$, and ABCG2 in the GBM-PDCL cells. Paired tumor samples were analyzed using the LightCycler Probe Master mix 2X (Roche, 04887301001) and the UPL detection system (Roche, 04483433001; primers are listed in supplementary table 1) in a Light Cycler 96 (Roche). For each qPCR, we made a duplicate measure in each experiment and we ran three distinct experiments at three different passages. PPIA expression was used for normalization. qPCR was used to investigate expression levels of $A B C A 1, A B C A 4, A B C A 13, A B C C 1, A B B C C 12, A B C C 13$, and ABCG1 in the validation cohort of GBM patients were performed in a 1534 wells plate using a Sybr green detection system in a Light Cycler 1534 (Roche). PPIA and PPIB expression were used for normalization. 
Immunohistochemistry

Formalin-fixed, paraffin-embedded (FFPE) tumor tissue sections were deparaffinized, rehydrated, and incubated in citrate buffer at $\mathrm{pH} 4.0$ for epitope retrieval in a microwave at 400W for 15 minutes. Blockade solution was made using PBS 0.1M supplemented with $0.4 \%$ triton, 3\% BSA and 10\% donkey serum. Anti-human ABCG2 antibody (BXP-21; Euromedex) and anti-human CD31 antibody (SP38; Thermo Fischer Scientific) were diluted at 1:100 in the blockade solution and incubated on the tissue slices overnight at $4^{\circ} \mathrm{C}$. Secondary antibodies conjugated with Alexa 488 and 447 were incubated on the slices for $2 \mathrm{~h}$ at room temperature. The slices were then mounted using fluoromount, supplemented with DAPI (0100-20; Clinisciences) and visualized using a microscope (Apotome Axio M2, Zeiss).

\section{Statistical analysis}

Comparison between $A B C$ transporter expression in GBM-PDCL and tumors was performed by a paired t-test with a Bonferroni correction using R.

RT-qPCR data bar charts were plotted with GraphPad PRISM 4, and analyzed by a two-way ANOVA test.

For analysis of OS and PFS, patients were divided in two groups from each side of the median. Kaplan Meier survival curves were made on GraphPad PRISM 4. Survivals of groups with "low" versus "high" expression were compared using Logrank (Mantel-Cox) test. Additional Cox test for survival in a multivariate modality was performed with $\mathrm{R}$ to assess the impact of MGMT promoter methylation status after a log2 transformation of $A B C A 13$ expression. 


\section{Results}

Expression of $A B C$ transporters in the commercial U251 GBM cell line, in GBM-PDCL and paired-GBM tumors

Out of the 49 ABC transporters, 19 are measurably expressed in U251 GBM cell lines versus $42 / 49$ in the GBM-PDCL line [8]. This highlights that GBM cell lines may lose $A B C$ transporter expression over time in adherent culture conditions including bovine fetal serum (Fig. 1, Panel A). When cultured in a differentiating medium containing fetal calf serum, the N13-1520 PDCL shows a slight non-significant trend for increased expression of $A B C B 1$ in RT-qPCR (Fig. 1C) associated with higher resistance to temozolomide in an in vitro viability assay $(p<0.0001)$ (Fig. 1D). These results suggest that the expression loss of most of the $A B C$ transporters may be due to the high passages after cell line establishment rather than exposure to fetal calf serum. It also highlights the importance of using a non-differentiating serum-free medium.

We already showed that, overall, GBM-PDCL are molecularly very close to their parental human tumors in a multi-omics analysis when cultured in a nondifferentiating serum-free medium [11]. We analyzed the same data set (i.e. 9 GBMPDCL and their tumor of origin) with a specific focus on expression levels of the 49 $A B C$ transporters acquired using Affymetrix ${ }^{\circledR}$ expression profiling microarray and RNA Seq platforms. Three ABC transporters were differentially expressed between the GBM-PDCL and their parental tumor in both transcriptomes (i.e. Affymetrix and RNA Seq): ABCB1, ABCG1 and ABCG2 (Figures 1B, 2A and 2B, and Table 1).

RT-qPCR validated the difference of expression for the three transporters (Figs. 2C, 2D and 2E; Supp. Fig. 1). 
$A B C$ transporters differentially expressed in GBM-PDCL compared to their parental tumors are expressed in the microenvironnement

We hypothesized that $A B C$ transporters are differentially expressed between GBMPDCL and their parental tumors (i.e. underexpression in GBM-PDCL versus paired human tumors) due to the expression by microenvironmental cells present in bulk tumor. We questioned http://web.stanford.edu/group/barres_lab/brain_rnaseq.html for expression of $A B C B 1, A B C G 1$ and $A B C G 2$ in normal murine cells [13]. $A B C B 1$, ABCG1 and ABCG2 are expressed by endothelial cells supporting our hypothesis. ABCG1 and ABCG2 are also expressed in additional cell types (i.e. astrocytes, neurons, oligodendrocytes) (Figs. 2E, 2F and 2G). Indeed, ABCG2 and CD31 expression are colocalized using immunohistochemistry in a human GBM (Fig. 2H).

\section{Clinical value of $A B C$ transporter expression in GBM patients}

We then investigated the prognostic value of the 49 ABC transporters in the 2013 TCGA GBM cohort. An overview of the expression of these transporters is presented in supplementary Fig. 2. Kaplan-Meier analysis highlighted an unfavorable prognostic impact of: (i) over-expression of $A B C A 1, A B C A 4, A B C C 12$ and $A B C G 1$ on OS, and (ii) over-expression of $A B C A 13, A B C C 1$ and $A B C C 13$ on PFS (Table 2).

To validate this finding, expression of $A B C A 1, A B C A 4, A B C A 13, A B C C 1, A B C C 12$, $A B C C 13$ and $A B C G 1$ was assessed in a homogeneously treated cohort of 51 newly diagnosed GBM patients (Fig. 3A). Only overexpression of ABCA13 was validated as negative prognostic factor in terms of PFS ( $p=0.004$, Figure 3B) and OS in univariate analysis ( $p=0.047$, Figure $3 C$ ). In multivariate analysis, $A B C A 13$ overexpression was found to be an independent poor prognostic factor of PFS $(p=0.05$ for $A B C A 13, H R=$ 
1.12) when MGMT promoter methylation status ( $p=0.002$ for $M G M T, H R=3.13$ ) is included in the statistical model, but not for OS. 


\section{Discussion}

GBM is a challenging cancer to treat. Indeed, GBM cells are highly resistant to treatments. The blood-brain and blood-tumor barriers limit the efficacy of drug treatments in GBM patients and are a major factor in primary resistance of GBM cells to chemotherapy.

The blood-brain and blood-tumor barriers are physical and biological barriers. ABC transporters play a pivotal role in the biology of both barriers. Indeed, they can expel chemotherapeutic agents from the brain tissue to the bloodstream [2]. Expression of $A B C$ transporters by the tumor cells themselves can also be responsible for tumor resistance to chemotherapies. Therefore, in the current work we have investigated their expression in GBM cell lines used for preclinical investigations of new therapeutic compounds and in human tumors.

Commercial GBM cell lines lack expression of most ABC transporters. Additional in vitro models that better recapitulate $A B C$ transporter expression patterns of human tumors must be also used before moving drugs to in vivo investigations. GBM-PDCL is an important tool expressing a larger number of $A B C$ transporters expressed in human GBM. However, addition of serum in the culture medium appeared to change expression patterns of $A B C$ transporters. A trend to the increased expression of $A B C B 1$ and an increased resistance to temozolomide in a serum-complemented medium is consistent with the fact that temozolomide is a substrate of ABCB1 [14]. Together, our data suggest that the efficacy of chemotherapeutic agents might be changed in vitro, if they are substrates of efflux pumps not expressed in the cell line used and whose expression can be modulated by fetal calf serum. Using GBM-PDCL closer to human GBM may reduce this overestimation and may be more relevant. 
To identify $A B C$ transporters impacting response to the standard of care of newly diagnosed GBM patients, we have correlated prognosis of GBM patients to ABC transporter expression in a training dataset (i.e. TCGA) and a validation dataset (i.e. personal cohort). ABCA13 expression was found to be a prognostic factor for PFS and $\mathrm{OS}$ in univariate analyses. Interestingly, based on multivariate analysis, ABCA13 expression provides additional prognostic information, in terms of PFS, to MGMT promoter methylation in young patients ( $<70$ years), in good clinical condition (KPS $>70 \%$ ) suffering from newly diagnosed GBM and treated with the standard of care. This supports for the first time ABCA13 expression as an independent prognostic biomarker in newly diagnosed GBM patients. Additional prospective and functional studies are warranted to investigate our findings further.

Little is known about ABCA13. ABCA13 is a large protein of 5058 amino acids encoded by $A B C A 13$ located on chromosome region $7 \mathrm{p} 12.3$ that is often polysomic in GBM. ABCA13 is highly expressed in the bone marrow, but not in normal brain [9]. Therefore, in GBM, ABCA13 is likely to be primarily expressed by tumor cells rather than microenvironmental cells. The molecular function of ABCA13 remains largely unknown [15]. However, ABCA13 has been linked to multiple human diseases, including cancer. ABCA13 genetic polymorphisms have been associated with psychiatric disorders [16-21] and success of coronary artery bypass surgery [22]. ABCA13 expression has also been linked to outcome in various cancers. In line with our study, ABCA13 overexpression is associated with poor prognosis in renal cell carcinoma [23], in gastric adenocarcinoma [24], and in ovarian serous carcinoma [25]. In contrast, ABCA13 overexpression is associated with improved outcomes in breast cancer [26] and in colorectal cancer [27]. Yun et al. reported an association of ABCA13 and stem-like phenotype in the "side population" of metastatic renal cell 
carcinoma. Overexpression of ABCA13 was also correlated with drug resistance, suggesting a potential role in drug efflux [23].

Overall our study suggests that $A B C$ binding cassette transporters, particularly $A B C A 13$, are important in both preclinical and clinical research in GBM. We have found that $A B C A 13$ is a potential prognostic factor in GBM patients and a putative actor of therapy resistance.Further studies are warranted to support our findings and to better understand the role of $A B C A 13$.

\section{Acknowledgments}

Institut Universitaire de Cancérologie

Fondation ARC pour la recherche sur le cancer

Association pour la Recherche sur les Tumeurs Cérébrales

Dr Michael Canney.

OncoNeuroTek tissue bank, Paris.

\section{Compliance with Ethical Standards}

This study was funded by the Fondation ARC pour la recherche sur le cancer and the Association pour la Recherche sur les Tumeurs Cérébrales.

All authors declare they have no conflict of interest with the present study.

All procedures performed in studies involving human participants were in accordance with the ethical standards of the institutional and/or national research committee and with the 1964 Helsinki declaration and its later amendments or comparable ethical standards. Informed consent was obtained from participants included in the study. 


\section{References}

1. Ostrom QT, Bauchet L, Davis FG, et al (2014) The epidemiology of glioma in adults: a "state of the science" review. Neuro-Oncol 16:896-913 . doi: 10.1093/neuonc/nou087

2. Dréan A, Goldwirt L, Verreault $M$, et al (2016) Blood-brain barrier, cytotoxic chemotherapies and glioblastoma. Expert Rev Neurother 1-16 . doi: $10.1080 / 14737175.2016 .1202761$

3. Vasiliou V, Vasiliou K, Nebert DW (2009) Human ATP-binding cassette (ABC) transporter family. Hum Genomics 3:281-290

4. Hartz AMS, Bauer B (2011) ABC transporters in the CNS - an inventory. Curr Pharm Biotechnol 12:656-673

5. Sparreboom A, Danesi R, Ando Y, et al (2003) Pharmacogenomics of ABC transporters and its role in cancer chemotherapy. Drug Resist Updat Rev Comment Antimicrob Anticancer Chemother 6:71-84

6. Khamisipour G, Jadidi-Niaragh F, Jahromi AS, et al (2016) Mechanisms of tumor cell resistance to the current targeted-therapy agents. Tumour Biol J Int Soc Oncodevelopmental Biol Med 37:10021-10039 . doi: 10.1007/s13277-016-50591

7. Brennan CW, Verhaak RGW, McKenna A, et al (2013) The somatic genomic landscape of glioblastoma. Cell 155:462-477 . doi: 10.1016/j.cell.2013.09.034

8. Lundberg E, Fagerberg L, Klevebring D, et al (2010) Defining the transcriptome and proteome in three functionally different human cell lines. Mol Syst Biol 6:450 . doi: $10.1038 / \mathrm{msb} .2010 .106$

9. Uhlén M, Hallström BM, Lindskog C, et al (2016) Transcriptomics resources of human tissues and organs. Mol Syst Biol 12:862

10. Stupp R, Mason WP, van den Bent MJ, et al (2005) Radiotherapy plus concomitant and adjuvant temozolomide for glioblastoma. $\mathrm{N}$ Engl $\mathrm{J}$ Med 352:987-996 . doi: 10.1056/NEJMoa043330

11. Rosenberg S, Verreault M, Schmitt C, et al (2016) Multi-omics analysis of primary glioblastoma cell lines shows recapitulation of pivotal molecular features of parental tumors. Neuro-Oncol. doi: 10.1093/neuonc/now160

12. Kamoun A, Idbaih A, Dehais C, et al (2016) Integrated multi-omics analysis of oligodendroglial tumours identifies three subgroups of $1 p / 19 q$ co-deleted gliomas. Nat Commun 7:11263 . doi: 10.1038/ncomms11263

13. Zhang $\mathrm{Y}$, Chen $\mathrm{K}$, Sloan SA, et al (2014) An RNA-sequencing transcriptome and splicing database of glia, neurons, and vascular cells of the cerebral cortex. $J$ Neurosci Off J Soc Neurosci 34:11929-11947 . doi: 10.1523/JNEUROSCI.186014.2014 
14. Goldwirt L, Beccaria K, Carpentier A, et al (2014) Irinotecan and temozolomide brain distribution: a focus on ABCB1. Cancer Chemother Pharmacol 74:185-193 . doi: 10.1007/s00280-014-2490-0

15. Tomioka M, Toda Y, Kurisu J, et al (2012) The effects of neurological disorderrelated codon variations of $A B C A 13$ on the function of the $A B C$ protein. Biosci Biotechnol Biochem 76:2289-2293 . doi: 10.1271/bbb.120563

16. Knight HM, Pickard BS, Maclean A, et al (2009) A cytogenetic abnormality and rare coding variants identify $\mathrm{ABCA} 13$ as a candidate gene in schizophrenia, bipolar disorder, and depression. Am J Hum Genet 85:833-846 . doi: 10.1016/j.ajhg.2009.11.003

17. Dwyer $\mathrm{S}$, Williams $\mathrm{H}$, Jones I, et al (2011) Investigation of rare non-synonymous variants at ABCA13 in schizophrenia and bipolar disorder. Mol Psychiatry 16:790-791 . doi: 10.1038/mp.2011.2

18. Pickard BS, Van Den Bossche MJA, Malloy MP, et al (2012) Multiplex amplicon quantification screening the $\mathrm{ABCA} 13$ gene for copy number variation in schizophrenia and bipolar disorder. Psychiatr Genet 22:269-270 . doi: 10.1097/YPG.0b013e32835185b3

19. Degenhardt F, Priebe L, Strohmaier J, et al (2013) No evidence for an involvement of copy number variation in ABCA13 in schizophrenia, bipolar disorder, or major depressive disorder. Psychiatr Genet 23:45-46 . doi: 10.1097/YPG.0b013e328358645b

20. Ma J, Lan X, Gao N, et al (2013) A genetic association study between common variants in the ABCA13 gene and schizophrenia in a Han Chinese population. Psychiatry Res 209:748-749 . doi: 10.1016/j.psychres.2013.07.013

21. Chen J, Khan RAW, Wang M, et al (2016) Association between the variability of the ABCA13 gene and the risk of major depressive disorder and schizophrenia in the Han Chinese population. World J Biol Psychiatry Off J World Fed Soc Biol Psychiatry 1-7 . doi: 10.1080/15622975.2016.1245442

22. Shah AA, Haynes C, Craig DM, et al (2015) Genetic variants associated with vein graft stenosis after coronary artery bypass grafting. Heart Surg Forum 18:E1-5

23. Yun E-J, Zhou J, Lin C-J, et al (2017) The network of DAB2IP-miR-138 in regulating drug resistance of renal cell carcinoma associated with stem-like phenotypes. Oncotarget. doi: 10.18632/oncotarget.17756

24. Araújo TM, Seabra AD, Lima EM, et al (2016) Recurrent amplification of RTEL1 and ABCA13 and its synergistic effect associated with clinicopathological data of gastric adenocarcinoma. Mol Cytogenet 9:52 . doi: 10.1186/s13039-016-0260-x

25. Nymoen DA, Holth A, Hetland Falkenthal TE, et al (2015) CIAPIN1 and ABCA13 are markers of poor survival in metastatic ovarian serous carcinoma. Mol Cancer 14:44 . doi: 10.1186/s12943-015-0317-1 
26. Hlaváč V, Brynychová $V$, Václavíková R, et al (2013) The expression profile of ATP-binding cassette transporter genes in breast carcinoma. Pharmacogenomics 14:515-529 . doi: 10.2217/pgs.13.26

27. Hlavata I, Mohelnikova-Duchonova B, Vaclavikova R, et al (2012) The role of $A B C$ transporters in progression and clinical outcome of colorectal cancer. Mutagenesis 27:187-196 doi: $10.1093 /$ mutage/ger075 


\section{Figures, tables and legends}

Figure 1
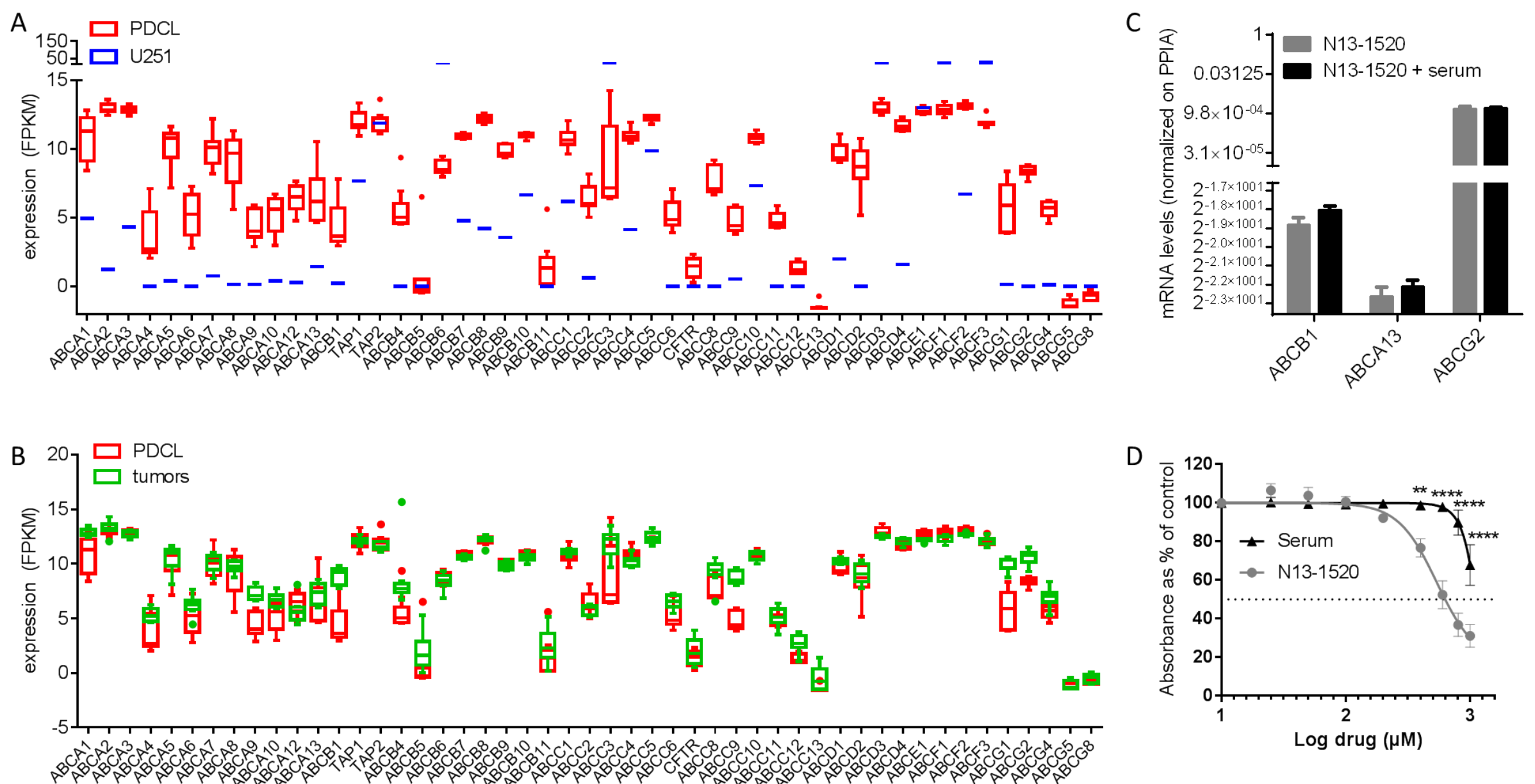
Figure 1: mRNA expression levels of the $49 A B C$ transporters. Panel A: Expression of the $A B C$ transporters in nine previously characterized GBM-PDCL and the adherent, serum-cultured human GBM cell line U251 MG. U251 MG transcriptome was extracted from published data [8]. Panel B: Expression of the ABC transporters in the nine GBM-PDCL and their parental tumors. Panel C: Expression of $A B C B 1, A B C A 13$ and $A B C G 2$ in the N13-1520 PDCL grown in its serum-free versus serum-complemented medium measured by RT-qPCR (T-test: $p=0.0620$ for ABCB1; NS for each couples ; error bars are in SD). Panel D: viability test of the N131520 PDCL grown in serum-free and serum-complemented medium after exposure to temozolomide $(p<0.0001$; error bars are in SEM). 

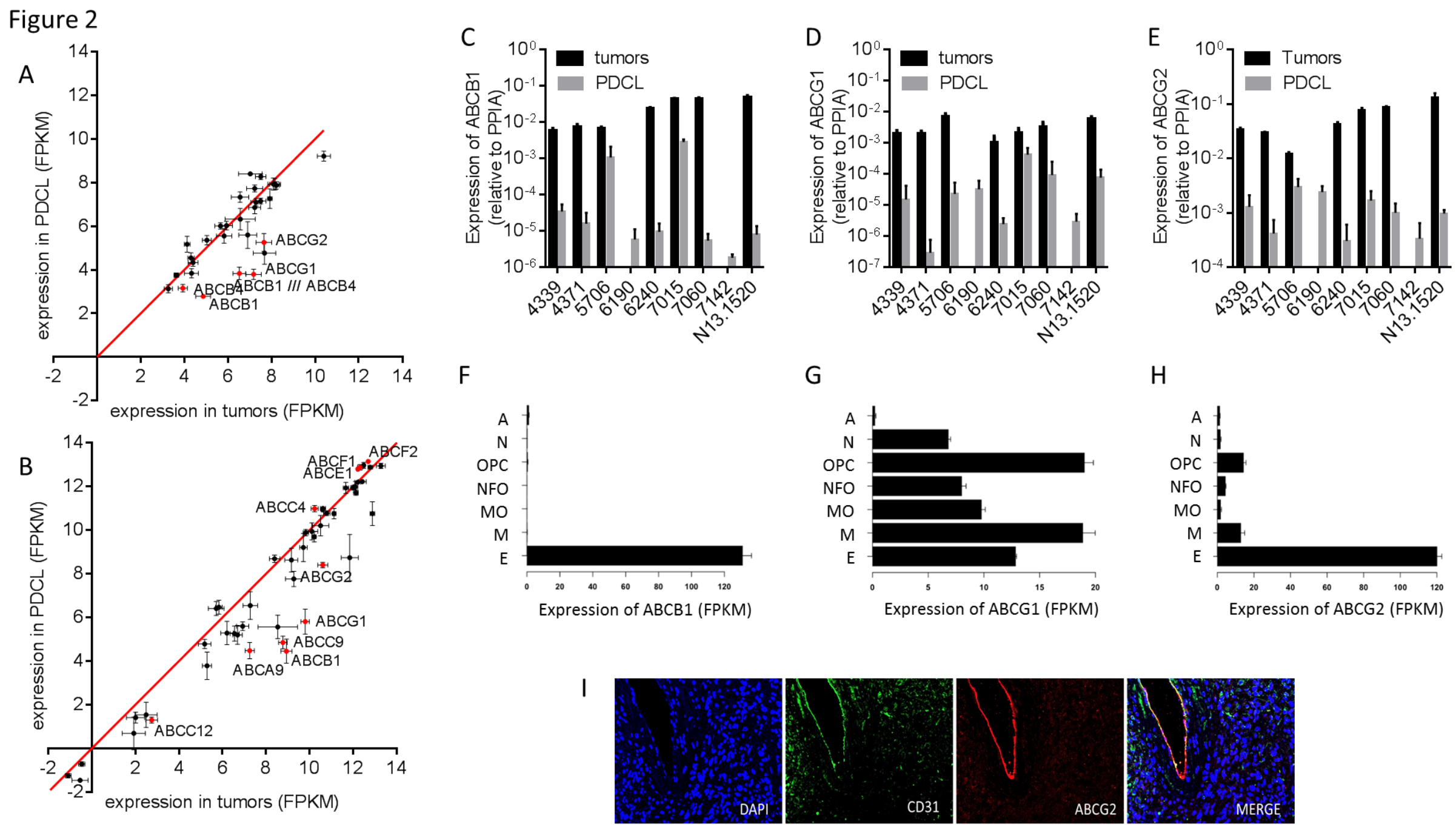
Figure 2: Correlation of mRNA expression levels of the $49 \mathrm{ABC}$ transporters in the nine GBM-PDCL versus their matched parental tumors. Panel A: Using transcriptome from Affymetrix expression profiling micro-arrays. Panel B: Using transcriptome from RNA sequencing. In both panels, red dots indicate the ABC transporters differentially expressed between the GBM-PDCL and their paired parental tumor in the paired t-test. Panels C, D and E represent QPCR data validating the expression of the three ABC transporters (i.e. ABCB1, ABCG1 and ABCG2 respectively) differentially, in both expression profiling platforms, expressed between GBM-PDCL and paired parental tumors. Tumor samples for the 4190 and the 7142 PDCL were ran out and could not be used for RT-qPCR. T-test PDCL vs tumor for ABCB1, ABCG1 and ABCG2 : $p<0.05$. Error bars are in SD. Bar charts in panels $F, G$ and $H$ represent the expression of $A B C B 1, A B C G 1$ and $A B C G 2$ respectively by the different cell types in the brain extracted from a database [9]. E : endothelial cells ; M : Microglia ; MO : myelinating oligodendrocytes ; NFO : Newly formed oligodendrocytes ; OPC : oligodendrocyte progenitor cells ; N : Neurons ; A : Astrocytes. Panel I shows colocalization of ABCG2 and CD31 in immunofluorescence in a human GBM. 
Figure 3
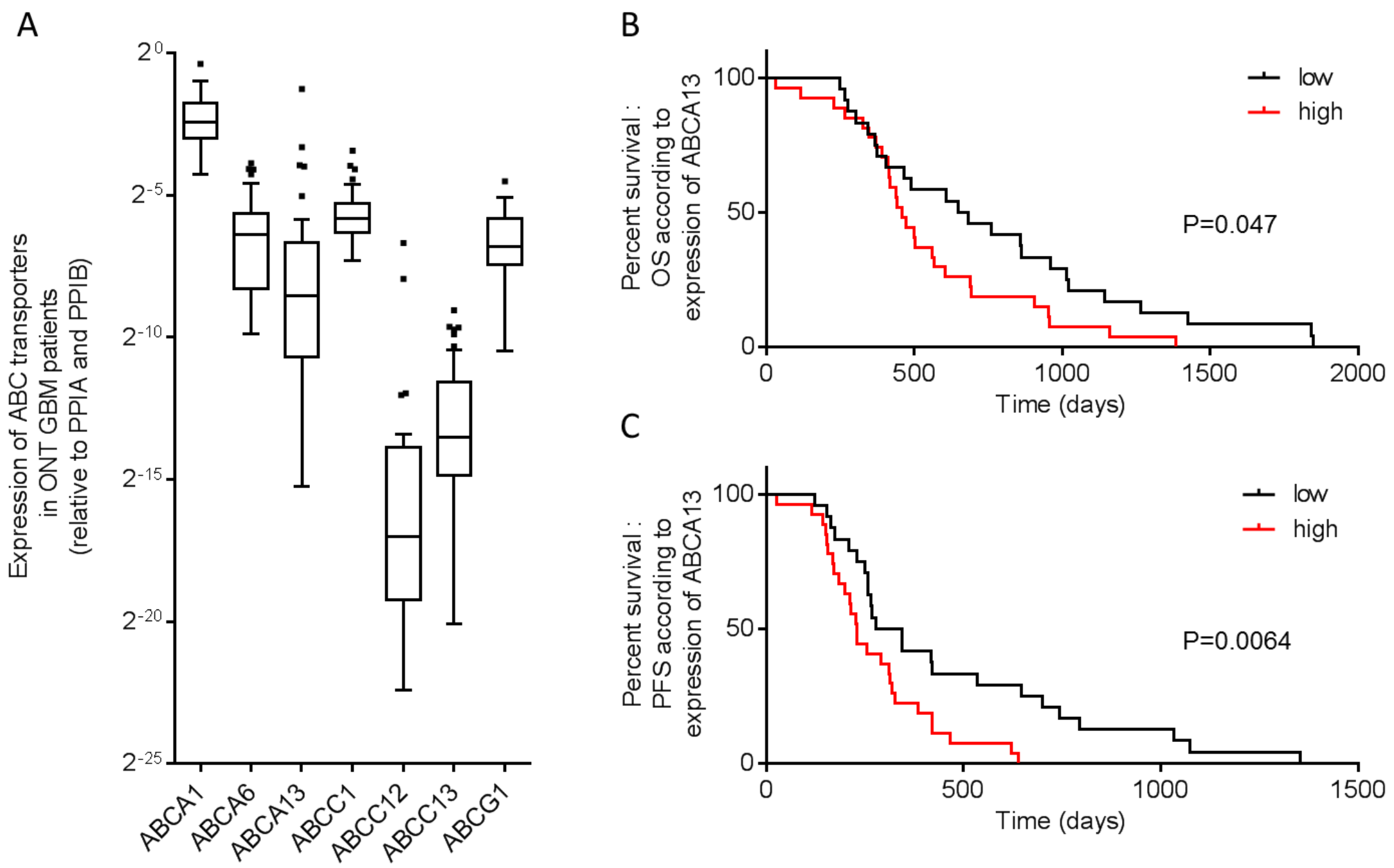

C

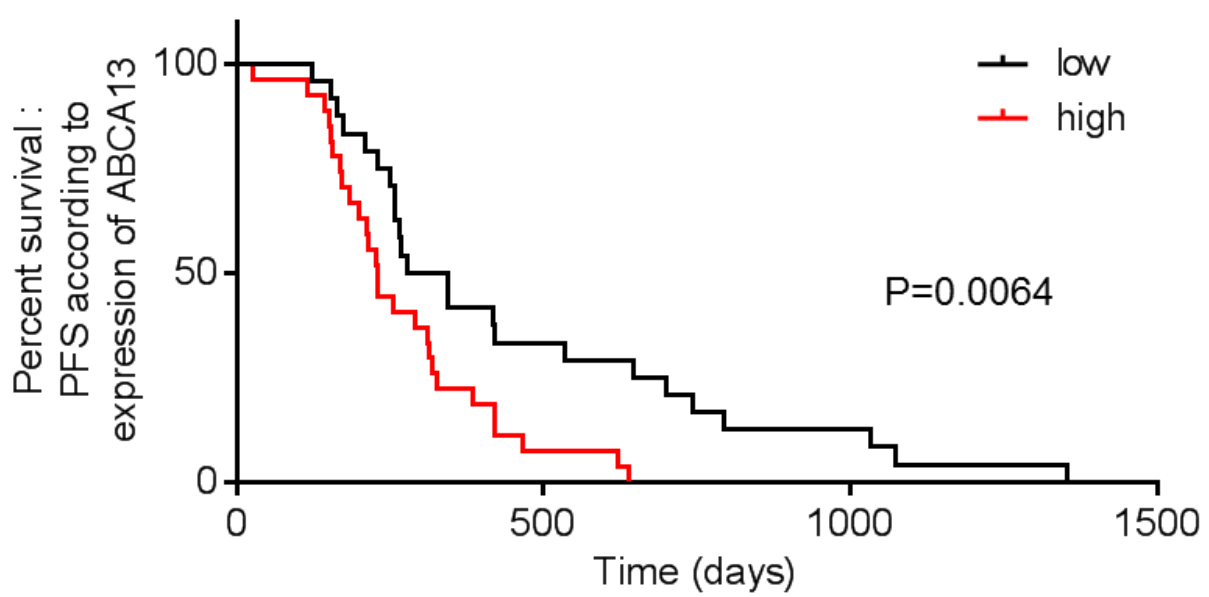


Figure 3: impact of $A B C A 1, A B C A 4, A B C A 13, A B C C 1, A B C C 12, A B C C 13$ and $A B C G 1$ in clinic. Panel $A$ : Expression of $A B C A 1$, ABCA4, ABCA13, ABCC1, ABCC12, ABCC13 and ABCG1 measured by RT-qPCR in the 51 GBM from OncoNeuroTek. Panel B: ABCA13 overexpression is significantly associated with shorter OS. Panel C: ABCA13 overexpression is significantly associated with shorter PFS. 


\section{Supplementary}

Figure 1
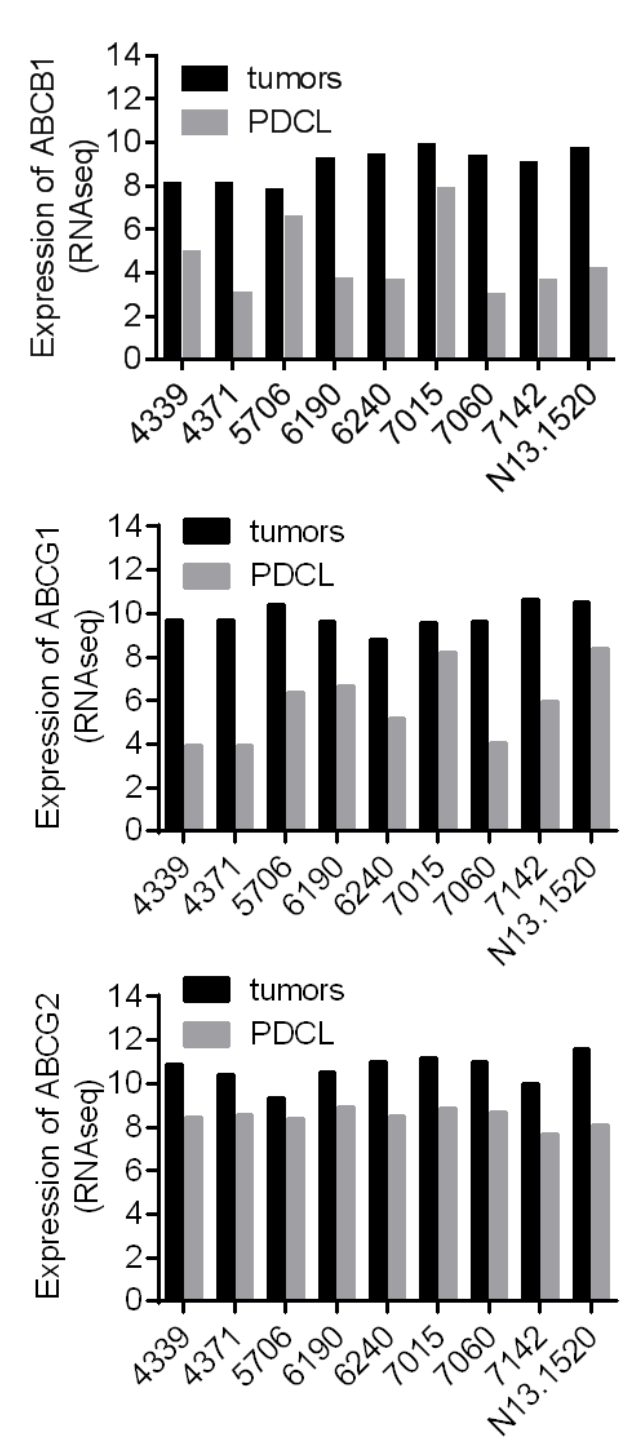
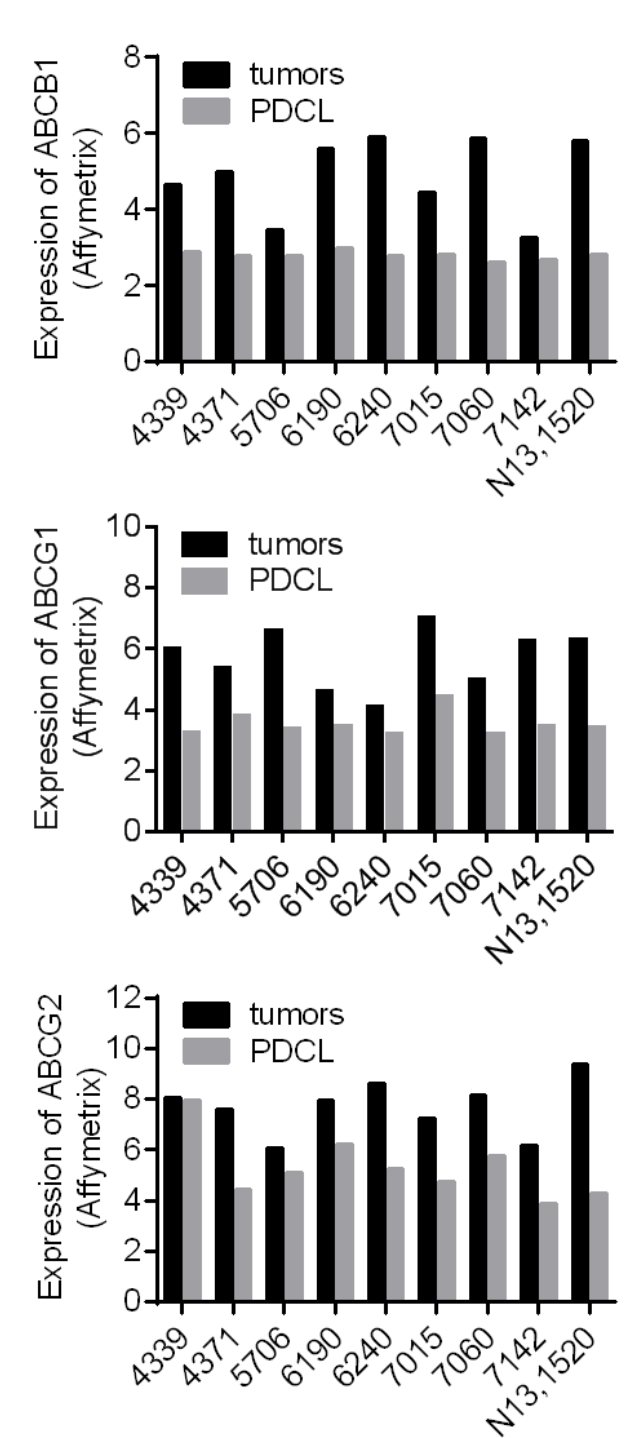
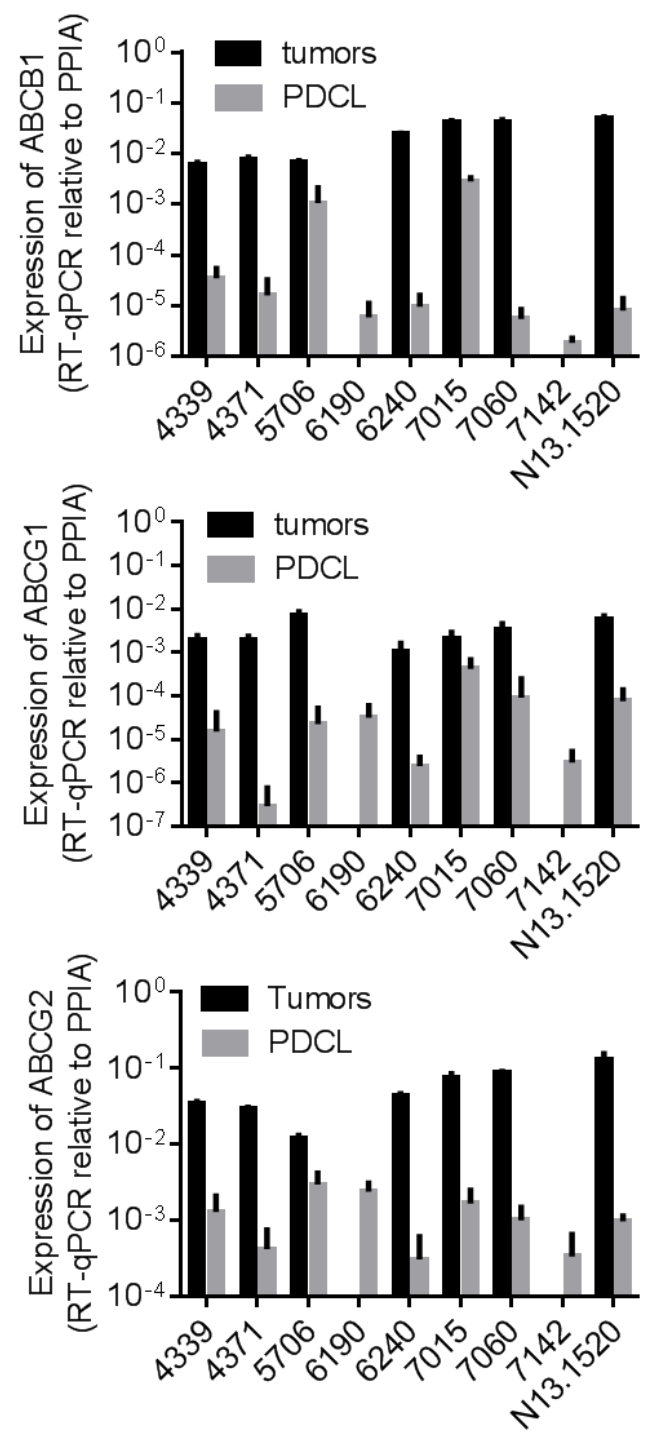
Supplementary Figure 1: mRNA expression levels of ABCB1, ABCG1 and ABCG2 (from top to bottom) in the 9 GBM-PDCL versus their paired parental tumors measured used RNAseq and Affymetrix microarray expression profiling and validated using qPCR (from left to right). RNAseq levels are closer to qPCR level compared to Affymetrix microarray expression profiling. Error bars are in SD. 


\section{Supplementary}

Figure 2

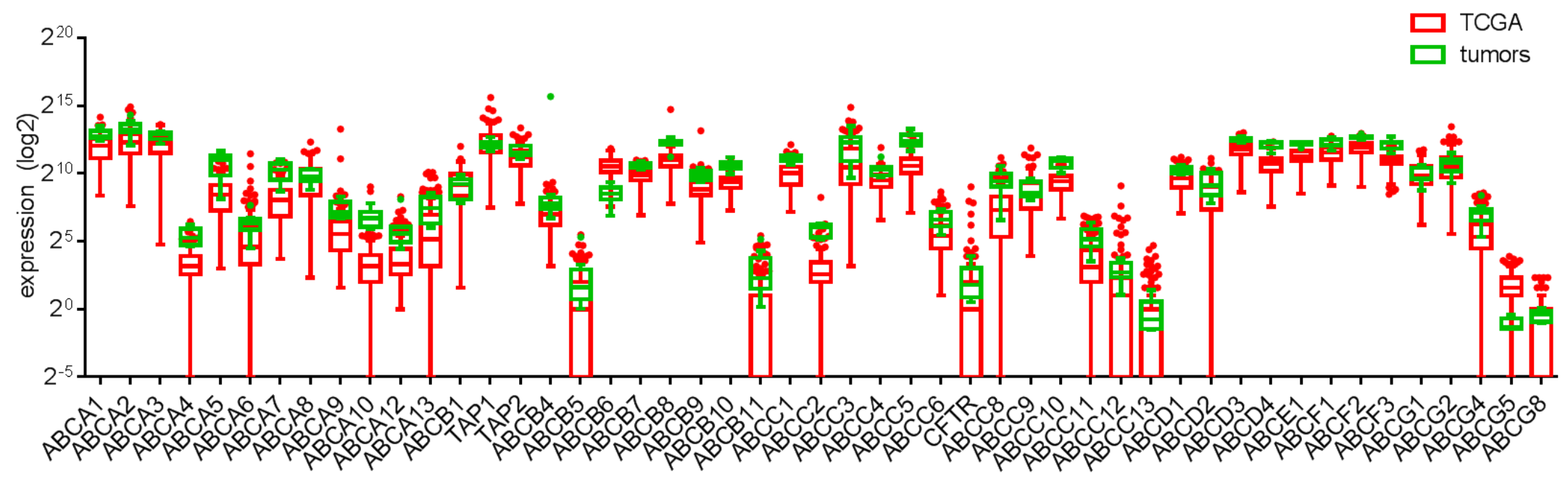

Supplementary Figure 2: mRNA expression levels of the 49 ABC transporters using RNA sequencing for the TCGA set of patients compared

the

9 tumors

corresponding

to

our

PDCL

presented

in

Figure

1. 
Table 1: Expression level of the ABC transporters in GBM-PDCL compared to their parental tumors using two gene expression profiling platforms (i.e. RNA-seq and Affymetrix gene expression profiling microarray)

\begin{tabular}{lcc}
\hline ADJUSTED P-VALUES & RNASEQ & AFFYMETRIX \\
\hline ABCB1 & 0.004 & 0.008 \\
ABCB1/ABCB4 & NA & 0.004 \\
ABCG1 & 0.004 & $<0.001$ \\
ABCG2 & 0.001 & 0.031 \\
\hline Legend : NA. not available in the design of the microarray. pval. $p$-value in the paired \\
t-test. $p$ adjusted.
\end{tabular}

Table 2: Prognostic value. on overall and progression free survival of expression level of the $49 \mathrm{ABC}$ transporters in glioblastoma patients from the TCGA dataset

\begin{tabular}{ccc}
\hline ABC TRANSPORTERS & OVERALL SURVIVAL & $\begin{array}{c}\text { PROGRESION FREE } \\
\text { SURVIVAL } \\
(\mathrm{p} \text {-value })\end{array}$ \\
\hline ABCA1 & 0.006 & $\mathrm{NS}$ \\
ABCA6 & 0.013 & $\mathrm{NS}$ \\
ABCA13 & $\mathrm{NS}$ & 0.014 \\
ABCC1 & $\mathrm{NS}$ & 0.031 \\
ABCC12 & 0.002 & $\mathrm{NS}$ \\
ABCC13 & $\mathrm{NS}$ & 0.012 \\
ABCG1 & 0.017 & $\mathrm{NS}$ \\
\hline
\end{tabular}

Legend: NS. not statistically significant 
Supplementary Table 1: qPCR primers and UPL probes

\begin{tabular}{|c|c|c|c|}
\hline GENE & PRIMER LEFT & PRIMER RIGHT & $\begin{array}{ll}\text { UPL } & \text { (IF } \\
\text { USED) } & \end{array}$ \\
\hline$A B C A 1$ & tgctgcatagtcttgggactc & acctcctgtcgcatgtcact & \\
\hline$A B C A 6$ & aaccatctacggggcatagacc & taacgactgcctggattgc & \\
\hline$A B C A 13$ & aagccctgctgtggaaga & ggccagaagaattcagcaag & Probe \#21 \\
\hline$A B C B 1$ & gaaatttagaagatctgatgtcaaaca & actgtaataataggcatacctggtca & Probe \#65 \\
\hline$A B C C 1$ & ccatgtgggaaaacacatctt & ctgtgcgtgaccaagatcc & \\
\hline$A B C C 12$ & gaggaagatgctggtataatcgtt & gagctggtgctcaggaactt & \\
\hline$A B C C 13$ & gcccactcataatgaagcaaa & cagagtttgcaaaaagactacaaca & \\
\hline$A B C G 1$ & tcagggacctttcctattcg & ttcctttcaggagggtcttgt & Probe \#22 \\
\hline$A B C G 2$ & tggcttagactcaagcacagc & tcgtccctgcttagacatcc & Probe \#56 \\
\hline
\end{tabular}

